The problem is seldom limited to the child alone and non-organic factors must also be addressed.

Aims: To audit the first 18 months of practice in a new regional EDS clinic from July 2007-December 2008

Methods: Retrospective chart review of clinic reports was carried out. All patients had formal assessment of EDS by a speech and language (SALT) and occupational therapist (OT), a medical review by a doctor and dietetics consult at each clinic visit.

Results: 28 patients received full assessment. Average age of $1^{\text {st }}$ consult was 4.16 years. $27 / 28$ patients had an underling diagnosis, the most common being; genetic disorder $(n=7)$, autism $(n=3)$, cerebral palsy $(n=3)$, Ex-prem $(n=3)$, developmental delay of unknown aetiology $(n=3)$. Diverse reasons for referral included(often $>1$ ); issues with coordination of swallow $(n=14)$, perceived behavioural issues $(n=6)$, chronic reflux $(n=5), P E G$ feeding $(n=4)$, aspiration( $n=4) .22 / 28$ patients had an abnormal EDS assessment. The two most common interventions initiated were a sensory feeding programme $(n=15)$ or food desensitisation programme $(n=4)$ by SALT/OT.

Conclusion: Our EDS clinic has provided further therapeutic intervention for children already with complex medical needs. In conjunction with medical therapy, behavioural and oromotor strategies can be applied successfully even in feeding disorders with an underlying organic cause.

1061

\section{EFFECTS OF BREASTFEEDING ON MORBIDITY AND DURATION OF HOSPITALIZATION OF CHILDREN WITH ACUTE DIARRHEA}

\author{
M. Berisha ${ }^{1,2}$, A. Maloku-Ceku ${ }^{1}$, R. Bejiqi ${ }^{1}$, \\ R. Retkoceri ${ }^{1}$ \\ ${ }^{1}$ Children's Clinic, ${ }^{2}$ University of Pristina, \\ Pristina, Albania
}

Introduction: The acute diarrhea is one of the most common causes of morbidity and for hospital treatment of children in nondeveloped countries. Etiology is usually viral infection but can be included other infective agents. Breastfeeding importance decreases morbidity, clinical manifestation and duration of hospitalization in compare with children with infant formulas.
Aim: of this presentation is to present the children hospitalized from acute diarrhea under 6 months old exclusively on breastfeeding with children of the same age feeded with infant formulas, in Children's Clinic.

Methods: Anamnestic data, clinical examination and statistical data.

Discussion: Retrospectively we analyzed 219 children under 6 month age hospitalized from acute diarrhea during 2008-2009. Children were divided in two groups depending on their feeding. In first group, were 66 children with breastfeeding, clinical manifestation and electrolyte disturbances in admission were less severe, hospital treatment duration was significantly shorter in compare with group of children without breastfeeding. None of children on the breastfeeding didn't presented clinical manifestations of severe dehydration in compare with second group were 21 children had severe dehydration.

Conclusion: Promotion of breastfeeding in nondeveloped countries, excluding other priority has very important role in prevention, severity and treatment of acute diarrhea.

Key word: Breastfeeding, Infant formula, Diarrhea, Hospitalisation duration.

1062

\section{THE SELECTED LIPIDS PARAMETERS IN NEWBORNS AND INFANTS}

E. Pac-Kozuchowska ${ }^{1}$, W. Furmaga-Jablonska ${ }^{2}$

${ }^{1}$ Department of Paediatric, ${ }^{2}$ Department of Neonates' and Infants' Pathology, Medical University of Lublin, Lublin, Poland

Introduction: Disorders of lipids metabolism, particularly elevated cholesterol concentration are favouring factors of atherosclerotic changes in the arteries. The lipids metabolism may be the results of the influence of genetic as well as environmental factors.

Aim: The aim of the present study was the evaluation of the concentration of triglycerides, total cholesterol and its fractions and apolipoproteins in the newborns and infants with relation to intrauterine growth, nutritional status and method of feeding from the birth. 